\title{
Minor Physical Anomalies and their Relationship to the Aetiology of Schizophrenia
}

KIERAN C. MURPHY and MICHAEL J. OWEN

\begin{abstract}
Background. Previous reports have suggested an increased prevalence of minor physical anomalies (MPAs) in schizophrenia.

Method. A review of the literature was performed to examine the relationship between MPAs, schizophrenia and other disorders.

Results. Previous studies demonstrate a number of methodological shortcomings. Moreover, MPAs are found in several other psychiatric disorders. Proponents of the neurodevelopmental model of schizophrenia have focused on an environmental explanation for the increased prevalence of MPAs. We argue that this may be overly simplistic and propose various ways in which genetic factors may also be involved.

Conclusions. Better studies should be performed to examine more precisely the nature of MPAs in schizophrenia and other disorders and also the relationship between MPAs and other developmental abnormalities. At present, it is unclear if MPAs are directly related to the pathogenesis of the disorder or even if they are related to the timing of critical events.
\end{abstract}

A possible association between physical abnormalities and mental illness was proposed as far back as 1896. Kraepelin, in one of his earliest descriptions of dementia praecox, reported that "so-called signs of degeneracy were often observed: smallness or deformity of the skull, child-like habitus, missing teeth, deformed ears"' (Kraepelin, 1896).

There has been a recent resurgence of interest in a possible association between what are now termed 'minor physical anomalies' (MPAs) and psychiatric illness. MPAs are slight anatomical defects of the head, hair, eyes, ears, mouth, hands and feet and are usually attributed to injury or atypical ectodermal differentiation during the first or second trimester of foetal life. An excess of MPAs in schizophrenia was first described systematically by Gualtieri et al (1982) and this finding has been replicated in the majority of subsequent studies (Guy et al, 1983; Green et al, 1989; O'Callaghan et al, 1991).

\section{Methodological issues}

Although there is impressive agreement on the increased prevalence of MPAs in schizophrenia, there are a number of methodological issues to consider. The majority of studies that have examined the prevalence of MPAs in schizophrenia have used the Waldrop scale, or a modification of it (Gualtieri et al, 1982; Guy et al, 1983; Green et al, 1989; O'Callaghan et al, 1991). This scale was devised 25 years ago and is based on abnormalities found in Down's syndrome (Waldrop et al, 1968). Many of the ratings are non-quantitative, for example, 'high' palate, 'abnormal' nails, and abnormalities are often expressed as a total score with little description of the specific abnormalities themselves. Although adequate inter-rater and test-retest reliability can be achieved, concern must be expressed about the lack of blindness to diagnosis when such measures are used on face-to-face examination, especially with non-patient control populations. As yet no convincingly blind study using photographs of patients and controls has been conducted. A definitive study should also be based upon more easily quantifiable measures and a wider range of morphometric data than those obtained by the Waldrop scale.

\section{Are minor physical anomalies specific to schizophrenia?}

The Waldrop scale has also been used to study MPAs in a variety of other psychiatric disorders. Campbell et al (1978) reported an excess in autistic children while Krouse \& Kauffman (1982) found that dysmorphology, in the form of an excessive number of MPAs, was the most frequently reported biological abnormality in attention deficit disorder.

There have been conflicting reports about the prevalence of MPAs in bipolar affective disorder. Lohr \& Flynn (1993) reported an increased prevalence of MPAs in bipolar disorder. In contrast, Green et al (1994b) found no significant differences between patients and normal controls, but the power of this study was low due to the small sample size. 
Consequently, an excess of MPAs does not appear to be specific to schizophrenia. This raises the question of whether the MPAs seen in schizophrenia differ from those seen in other disorders. Unfortunately, it is not possible to answer this adequately at present as most studies have presented data only in the form of total anomaly scores and the rates of individual anomalies are seldom described. This is probably because most studies to date have been based on relatively small samples and have lacked sufficient power to demonstrate statistically significant differences in individual anomalies.

\section{What is the aetiological significance of MPAs in schizophrenia?}

The increased prevalence of MPAs in schizophrenia has generally been interpreted as evidence that schizophrenia results from a neurodevelopmental abnormality occurring at some time during the first or second trimester of foetal growth (Mednick et al, 1988; Murray et al, 1992). However, determining more precisely when MPAs evolve has been difficult because the effect of an insult on development depends not only upon its timing but also on its severity (Nowakowski, 1987). In other words, the same minor physical anomaly could result from a relatively minor insult in early foetal development or a more marked insult later. A further problem arises because MPAs involve a large number of anatomic sites which develop in the foetus at somewhat different times (Nishimura, 1983).

Evidence implicating a first trimester insult has been provided by O'Callaghan et al (1991) who reported that schizophrenics with MPAs were significantly more likely to have a history of bleeding during the first trimester. Although foetal organogenesis is mostly completed in the first trimester, the second trimester is a critical period of massive neuronal migration from the periventricular germinal matrix to the cortex (Hamilton et al, 1972). Migration of the ectodermal cells of the foetal upper limb is simultaneous with neuronal migration to the cortex. In a study of monozygotic twins discordant for schizophrenia, Bracha et al (1991) reported that the affected co-twins had significantly higher total scores of fourth and fifth month dysmorphological hand anomalies compared with non-affected co-twins, implicating a second trimester insult.

The majority of proponents of the neurodevelopmental model have focused on an environmental rather than a genetic explanation for the excess of MPAs seen in schizophrenia (Mednick et al, 1988; Murray et al, 1992). Bleeding during the first trimester is the pregnancy complication most clearly associated with MPAs in schizophrenia (O'Callaghan et al, 1991), but this could equally be secondary to a pre-existing foetal abnormality rather than of primary aetiological significance. Additionally, although Mednick et al (1988) reported that viral infection in the second trimester was significantly associated with the subsequent development of schizophrenia, there is no evidence for an association between maternal influenza and the subsequent development of MPAs in schizophrenia.

Firestone et al (1978) have suggested a role for genetic factors in the aetiology of MPAs. Further evidence to support this hypothesis comes from the observation that similar physical anomalies can also result from chromosomal disorders such as Down's syndrome and trisomy 13 (Smith, 1976). One possibility is that the increased prevalence of MPAs may reflect the operation of the same aberrant genes responsible for disordered neurodevelopment in schizophrenia. This is certainly an attractive hypothesis as both the CNS and the anatomic structures involved in MPAs are ectodermally derived. O'Callaghan et al (1991) have reported that MPAs are particularly common in schizophrenics with a family history of the disorder, and this lends support to a genetic explanation.

The association of chromosomal disorders with MPAs is of interest as this suggests that schizophrenia may itself sometimes result from chromosomal abnormalities. Gross chromosomal anomalies have been documented in schizophrenia (Bassett, 1992) but are not seen in the majority of cases. However, it is possible that more subtle abnormalities below the resolution of classical cytogenetic techniques will be revealed by more sensitive molecular methods.

It may be overly simplistic to suppose that MPAs reflect only genetic or environmental factors alone and they may well result from the operation of both classes of agent. One possibility is that heterogeneity exists and MPAs are sometimes genetic and sometimes environmental in origin. A more attractive explanation is that, like schizophrenia, they reflect the combined operation of both classes of agent. This is to take an orthodox polygenic (or oligogenic) multifactorial threshold liability view of schizophrenia (McGuffin et al, 1994). According to this, MPAs share genetic and environmental factors with schizophrenia and both arise from the same pathogenic substrate such as a disorder of ectodermal development. This approach would accord well with the observation that MPAs are common in cases with a high loading of risk factors such as family history, pregnancy complications or in those with an early age of onset. If we accept this explanation, we are 
forced to accept one of two further conclusions. The first is that the MPAs seen in other disorders such as autism and hyperactivity have different aetiologies from those in schizophrenia and will perhaps appear quantitatively and qualitatively different when studied in sufficient detail. The second conclusion is that other disorders associated with MPAs share a common aetiological basis with schizophrenia. Some evidence for the latter conclusion comes from the observation that individuals with autism may be at greater risk for schizophrenia than was previously recognised (Petty et al, 1984).

\section{Developmental instability, fluctuating asymmetry, MPAs and schizophrenia}

There is increasing interest in the concepts of developmental instability, fluctuating asymmetry and their relationship to schizophrenia. Developmental stability is the ability of an organism to properly execute its ontogenetic programme despite adverse environmental conditions (Waddington, 1957). Fluctuating asymmetry (FAS) is described as random deviation from symmetry in a bilateral organism and is a useful measure of developmental stability. Measures of FAS reflect the degree to which an organism can buffer itself against a wide range of environmental insults and still develop according to its genetically determined potential (Markow, 1992).

Many phenotypic similarities have been reported among the autosomal trisomy syndromes (Taylor, 1968) and this observation led Shapiro (1975) to report that the MPAs seen in Down's syndrome represented a "generalized amplified developmental instability" which were a direct consequence of trisomy 21. More recently, Blum-Hoffmann et al (1988) provided further evidence to support this hypothesis of developmental instability in chromosomal aneuploidy.

There is some evidence that individuals with greater heterozygosity within a population have increased developmental stability (Leary et al, 1983). Consequently, the degree of developmental instability, as measured by FAS, is also an index of overall levels of homozygosity present within a population. There is an impressive body of evidence that patients with schizophrenia show greater FAS than normal controls (Markow \& Gottesman, 1989). This has been explained within the polygenic/ multifactorial view of schizophrenia because those whose liability exceeds the threshold for disease are likely to be homozygous at many of the polygenic loci conferring susceptibility to the disorder (Markow, 1992).
Recently, Green et al (1994a) have shown that MPAs in schizophrenia are associated with dermatoglyphic fluctuating asymmetry. This suggests that both MPAs and FAS may be related to the same underlying developmental instability. This explanation could also account for the lack of specificity of MPAs, as developmental instability would be expected in other polygenic (or oligogenic) disorders. According to this view, MPAs are not only epiphenomena but may be unrelated directly to the pathogenesis of the disorder. It may therefore be inappropriate to conclude that their occurrence supports the neurodevelopmental view of schizophrenia. Indeed, other so-called neurodevelopmental abnormalities in schizophrenia might be the result of developmental instability and may be similarly unrelated to the pathogenesis of the disorder. In this case, we would predict that these phenomena would be non-specific and associated with FAS as well as other measures of developmental instability.

\section{Conclusions}

A review of the literature suggests that studies which have examined the prevalence of MPAs in schizophrenia and other disorders demonstrate a number of methodological shortcomings. In future, studies should be blind, with a greater range of morphometric variables and the nature of the individual anomalies should be described. In addition, issues concerning the lack of specificity should be addressed by comparing MPAs in schizophrenia with those reported in other disorders.

We should avoid overly simplistic explanations of what MPAs can tell us about the aetiology of schizophrenia. A review of the current evidence suggests that even the assumption that they are related to the timing of critical events may be premature.

\section{Clinical implications}

- MPAs are common in schizophrenia.

- MPAs are commonly associated with a positive family history, obstetric complications and an early age of onset.

- Schizophrenic patients with dysmorphic features may have chromosomal abnormalities.

\section{Limitations}

- Numerous methodological shortcomings in previous studies.

- MPAs are not specific to schizophrenia.

- Relationship to the aetiology of schizophrenia is uncertain. 


\section{Acknowledgement}

This work was supported by Glaxo Research and Development.

\section{References}

BASst IT, A. S. (1992) Chromosomal aberrations and schizophrenia British Journal of Psychiatry, 161, 323-334.

Bi um-Hoffmann, E., Rehder, H. \& Langenbeck, U. (1988) Skeletal anomalies in trisomy 21 as an example of amplified developmental instability in chromosome disorders: A histological study of the feet of 21 mid-trimester fetuses with trisomy 21. American Journal of Medical Genetics, 29, 155-160.

Bracha, H. S., Torrey, E. F., Bigelow, L. B., et al (1991) Subtle signs of prenatal maldevelopment of the hand ectoderm in schizophrenia: A preliminary monozygotic twin study. Biological Psychiatry, 30, 719-725.

Campbelt, M., Gelller, B., Small, A. M., et al (1978) Minor physical anomalies in young psychotic children. American Journal of Psychiatry 135, 573-575.

Firestone, P., Peters, S., Riviere, M., et al (1978) Minor physical anomalies in hyperactive, retarded and normal children and their families. Journal of Child Psychology and Psychiatry, 19, $155-160$.

Gritin, M. F., Satz, P., Galer, D. J., et al (1989) Minor physical anomalies in schizophrenia. Schizophrenia Bulletin, 15, 91-99.

- Bracha, H. S., SATz, P., et al (1994a) Preliminary evidence for an association between minor physical anomalies and second trimester neurodevelopment in schizophrenia. Psychiatry Research, 53, 119-127.

- Satz, P. \& Christenson, C. (1994b) Minor physical anomalies in schizophrenia patients, bipolar patients, and their siblings. Schizophrenia Bulletin, 20, 433-440.

Gual mierı, C. T., Adams, A., Shen, C. D., et al (1982) Minor physical anomalies in alcoholic and schizophrenic adults and hyperactive and autistic children. American Journal of Psychiatry, 139, 640-643.

GuY, J. D., Majorski, L. V., Wal.tace, C. J., et al (1983) The incidence of minor physical anomalies in adult male schizophrenics. Schizophrenia Bulletin, 9, 571-582.

Hamilton, W. J., Boyd, J. D. \& Mossman, H. W. (1972) Human Embryology: Prenatal Development of Form and Function. Cambridge: Heffer.

Kratpelin, E. (1896) Dementia praecox. Psychiatrie (5th edn). Leipzig: Barth.
Krouse, J. P. \& Kauffmann, J. M. (1982) Minor physical anomalies in exceptional children: $A$ review and critique of research. Journal of Abnormal Child Psychology, 10, 247-264.

Leary, R. F., Allendorf, F. W. \& Knudsen, K. L. (1983) Developmental stability and enzyme heterozygosity in rainbow trout. Nature, 301, 71-72.

LOHR, J. B. \& FLYNN, K. (1993) Minor physical anomalies in schizophrenia and mood disorders. Schizophrenia Bulletin, 19. $551-556$.

Markow, T. A. (1992) Genetics and developmental stability: an integrative conjecture on aetiology and neurobiology of schizophrenia. Psychological Medicine, 22, 295-305.

— \& Gottesman, I. I. (1989) Dermatoglyphic fluctuating asymmetry in psychotic twins. Psychiatry Research, 29, 37-43.

MCGuffin, P. OWen, M. J., O'Donovan, M. C., et al (1994) Schizophrenia. In Seminars in Psychiatric Genetics, pp. 87-109. London: Gaskell.

Mednick, S. A., Machon, R. A., Huttunen, M. O., et al (1988) Adult schizophrenia following prenatal exposure to an influenza epidemic. Archives of General Psychiatry, 45, 189-192.

Murray, R. M., O'Callaghan, E., Castle, D. J., et al (1992) A neurodevelopmental approach to the classification of schizophrenia. Schizophrenia Bulletin, 18, 319-332.

Nishimura, H. (1983) Atlas of Human Prenatal Histology. New York: Igaku-Shoin.

NowakowSKI, R. S. (1987) Basic concepts of CNS development. Child Development, 58, 568-595.

O'Callaghan, E., Larkin, C., Kinsella, A., et al (1991) Familial, obstetric and other clinical correlates of minor physical anomalies in schizophrenia. American Journal of Psychiatry, 148, 479-483.

Petty, L. K., Ornitz, E. M., Michelman, J. D., et al (1984) Autistic children who become schizophrenic. Archives of General Psychiatry, 41, 129-135.

Shapiro, B. L. (1975) Amplified developmental instability in Down's syndrome. Annals of Human Genetics, 38, 429-437.

Smith, D. W. (1976) Recognisable Patterns of Human Malformation: Genetic, Embryologic and Clinical Aspects. Philadelphia: W. B. Saunders.

TAYLOR, A. I. (1968) Autosomal trisomy syndromes: a detailed study of 27 cases of Edwards' syndrome and 27 cases of Patau's syndrome. Journal of Medical Genetics, 5, 227-252.

Waddington, C. H. (1957) The Strategy of the Genes. New York: Macmillan.

Waldrop, M. F., Pederson, P. A. \& Bel.t, R. Q. (1968) Minor physical anomalies and behaviour in preschool children. Child Development, 39, 391-400.

Kieran Murphy, Department of Psychological Medicine; Michael J. Owen, Departments of Psychological Medicine and Medical Genetics, University of Wales College of Medicine

Correspondence: Dr K. Murphy, Department of Psychological Medicine, University of Wales College of Medicine, Heath Park, Cardiff CF4 4XN, Wales. Fax: 01222747839

(First received 21 March 1995, final revision 26 July, accepted 10 August 1995) 